\title{
Distributed Algebraic Connectivity Estimation for Undirected Graphs with Upper and Lower Bounds
}

\author{
Rosario Aragues d,f, Guodong Shi ${ }^{\mathrm{b}}$, Dimos V. Dimarogonas ${ }^{\mathrm{b}, \mathrm{c}}$, Carlos Sagues ${ }^{\mathrm{a}}$, \\ Karl H. Johansson ${ }^{b}$, Youcef Mezouar ${ }^{\mathrm{e}, \mathrm{f}}$ \\ a Instituto de Investigación en Ingeniería de Aragón, Universidad de Zaragoza, Spain \\ ${ }^{\mathrm{b}}$ Access Linnaeus Centre, Royal Institute of Technology (KTH), Sweden \\ ${ }^{\mathrm{c}}$ Centre of Autonomous Systems (CAS), Royal Institute of Technology (KTH), Sweden \\ ${ }^{\mathrm{d}}$ Clermont Université, Institut Pascal, BP 10448, F-63000 CLERMONT-FERRAND, France

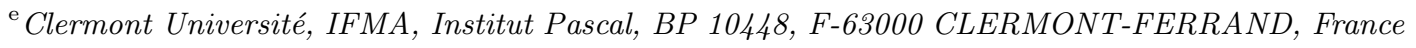 \\ ${ }^{\mathrm{f}}$ CNRS, UMR 6602, IP, F-63171 Aubière, France
}

\begin{abstract}
The algebraic connectivity of the graph Laplacian plays an essential role in various multi-agent control systems. In many cases a lower bound of this algebraic connectivity is necessary in order to achieve a certain performance. Lately, several methods based on distributed Power Iteration have been proposed for computing the algebraic connectivity of a symmetric Laplacian matrix. However, these methods cannot give any lower bound of the algebraic connectivity and their convergence rates are often unclear. In this paper, we present a distributed algorithm for estimating the algebraic connectivity for undirected graphs with symmetric Laplacian matrices. Our method relies on the distributed computation of the powers of the adjacency matrix and its main interest is that, at each iteration, agents obtain both upper and lower bounds for the true algebraic connectivity. It was proven that both bounds successively approach the true algebraic connectivity with the convergence speed no slower than $O(1 / k)$.
\end{abstract}

\section{Introduction}

Consensus problems are connected to diverse applications in multi-agent systems, including sensor fusion, flocking, formation control or rendezvous among others [10]. The algebraic connectivity $\lambda_{\star}(\mathcal{L})$, defined as the second smallest eigenvalue of the graph Laplacian $\mathcal{L}$, is an important network property for all the previous systems to reach convergence and it characterizes the convergence rate. Equivalently, the essential spectral radius $\rho_{\text {ess }}(\mathcal{W})$, defined as the second largest modulus eigen-

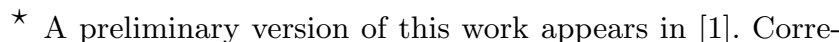
sponding author R. Aragues. Tel. +34-976762472. Fax +34976761914.

Email addresses: raragues@unizar es (Rosario Aragues), guodongs@kth.se (Guodong Shi), dimos@kth.se (Dimos V. Dimarogonas), csagues@unizar.es (Carlos Sagues), kallej@ee.kth.se (Karl H. Johansson), Youcef .MEZOUAR@ubpmes . univ-bpclermont.fr (Youcef Mezouar).
}

value of $\mathcal{W}$, plays an important role in systems that rely on stochastic weight matrices $\mathcal{W}$. These two problems are addressed in this paper.

Connectivity control methods establish agent motions that preserve or maximize some network connectivity property. Control laws for rendezvous and formation control that maintain the initial graph connectivity have been proposed in [7]. A similar problem has been addressed in [2], where in addition the control law is ensured to remain bounded. The $k$-connectivity matrix of the graph is computed in a centralized fashion in [21]. Several distributed methods compute spanning subgraphs [22], specific Laplacian eigenvectors [13], moments (mean, variance, skewness and kurtosis) of the Laplacian eigenvalue spectrum [12], or maximize the algebraic connectivity through motion control without actually computing it [16]. Although these control methods improve the network connectivity, they do not characterize any particular Laplacian eigenvalue, as we are going to propose. 
The Laplacian eigenvalues are estimated in [3] by making the agents execute a local interaction rule that makes their states oscillate at frequencies corresponding to these eigenvalues. Agents use the Fast Fourier Transform (FFT) on their states to identify these eigenvalues. The main limitations of this work are the nontrivial adjustment of the FFT, and the fact that some nodes may observe only a subset of the eigenvalues. The algebraic connectivity is computed in [9], by iteratively bisecting the interval where it is supposed to belong to. At each iteration, in order to check if the algebraic connectivity belongs to the upper or lower bisected intervals, a test function is executed for several steps.

Most of the remaining Laplacian spectra estimation solutions rely on the Power Iteration method or variations $[4,8,11,14,20]$. Power Iteration [6] selects an initial vector and then repeatedly multiplies it by a matrix and normalizes it. This vector converges to the eigenvector associated to the leading eigenvalue (the one with the largest absolute value) of the matrix. The original matrix can be previously deflated so that a particular eigenvalue becomes the leading one. Distributed Power Iteration approaches $[4,8,14,20]$ often execute, between consecutive steps, several iterations of methods with asymptotic convergence for the normalization of the vectors and the deflation of the Laplacian, e.g., average consensus. The use of these approximated, not exact, norms or deflation values, introduces errors in the system which are hard to analyze. A recent interesting approach that does not suffer from these problems is given in [11]; deflation is avoided by building a zero-mean initial vector, and normalization is computed with max-consensus, which converges in finite time.

Although some of the previous methods may ensure convergence [20], bounded estimation errors [14], or give some intuitions about the convergence rate [11], none of them truly provides an accurate characterization of the convergence speed and the relationship between the estimated and true algebraic connectivity values per iteration in the form of lower or upper bounds. For this reason, they must be compulsorily executed in a first phase previous to any algorithm that requires the knowledge of the algebraic connectivity. Instead, it is more interesting to execute both processes in parallel, and this can only be achieved if we accurately know how the estimates approach the true algebraic connectivity, i.e., if we characterize these bounds as we propose here.

In this paper, we show that Power Iteration methods give an upper bound of the algebraic connectivity. If we consider the estimated algebraic connectivity after a finite number of steps $k$, these methods believe that the network has a connectivity slightly higher than the true one, and thus their combination (execution in parallel) with higher level algorithms is not straightforward. We present an alternative distributed method for computing the algebraic connectivity (Section 3), whose main in- terest is that it provides upper and lower bounds for the true algebraic connectivity at each iteration. We prove that both bounds converge to the true algebraic connectivity, with a convergence speed no slower than $O(1 / k)$.

This paper is organized as follows: Section 2 states the problem; Section 3 presents our Distributed Algebraic Connectivity estimation method and discusses its benefits; Section 4 discusses how the method can be used for estimating the essential spectral radius of a weight matrix, instead of the Laplacian algebraic connectivity; Section 5 evaluates our method in a simulated scenario; and Section 6 states the conclusions.

\section{Preliminaries}

We use the notation defined in Table 1.

Consider a set of $n \in \mathbb{N}$ agents with indices $i \in$ $\{1, \ldots, n\}$. The agents can exchange information with nearby nodes. This information can be represented by an undirected graph $\mathcal{G}=(\mathcal{V}, \mathcal{E})$, where $\mathcal{V}=\{1, \ldots, n\}$ are the agents, and $\mathcal{E}$ are the edges. There is an edge $(i, j) \in \mathcal{E}$ between nodes $i$ and $j$ if they can exchange data. We say a $n \times n$ matrix $C$ is compatible with $\mathcal{G}$ if $C_{i j}=0$ iff $(i, j) \notin \mathcal{E}$ for $j \neq i$; note that we let the elements in the diagonal $C_{i i}$ be either equal or different than 0 . The adjacency matrix $\mathcal{A} \in\{0,1\}^{n \times n}$ of $\mathcal{G}$ is defined by

$$
\mathcal{A}_{i j}=1 \text { if }(i, j) \in \mathcal{E}, \mathcal{A}_{i j}=0 \text { otherwise, for } i, j \in \mathcal{V} .
$$

We assume that the undirected communication graph $\mathcal{G}$ is connected. We use $\mathcal{N}_{i}$ for the set of neighbors of a node $i$ with whom $i$ can exchange data, $\mathcal{N}_{i}=\{j \mid(i, j) \in \mathcal{E}\}$, and we let $d_{i}$ be the degree of node $i$ defined as the cardinality of $\mathcal{N}_{i}$, and $d_{\max }=\max _{i \in \mathcal{V}} d_{i}$.

The Laplacian matrix $\mathcal{L} \in \mathbb{R}^{n \times n}$ of $\mathcal{G}$ is the positivesemidefinite matrix

$$
\mathcal{L}=\operatorname{diag}(\mathcal{A} \mathbf{1})-\mathcal{A} .
$$

Note that both $\mathcal{A}$ and $\mathcal{L}$ are compatible with the graph. In this paper we sort the eigenvalues of $\mathcal{L}$ as follows,

$$
\lambda_{1}(\mathcal{L}) \leq \lambda_{2}(\mathcal{L}) \leq \cdots \leq \lambda_{n}(\mathcal{L})
$$

The Laplacian matrix $\mathcal{L}$ has the following well known properties, see e.g., [10]: (i) Its eigenvalues are upper bounded by $\lambda_{n}(\mathcal{L}) \leq 2 d_{\text {max }}$; (ii) It has an eigenvector $\mathbf{v}_{1}(\mathcal{L})=1 / \sqrt{n}$ with associated eigenvalue $\lambda_{1}(\mathcal{L})=0$, $\mathcal{L} \mathbf{1} / \sqrt{n}=\mathbf{0}$; and (iii) When the graph $\mathcal{G}$ is connected, then all the other eigenvalues are strictly greater than zero.

The algebraic connectivity of $\mathcal{G}$ denoted by $\lambda_{\star}(\mathcal{L})$ is the second-smallest eigenvalue $\lambda_{2}(\mathcal{L})$ of the Laplacian 
Table 1

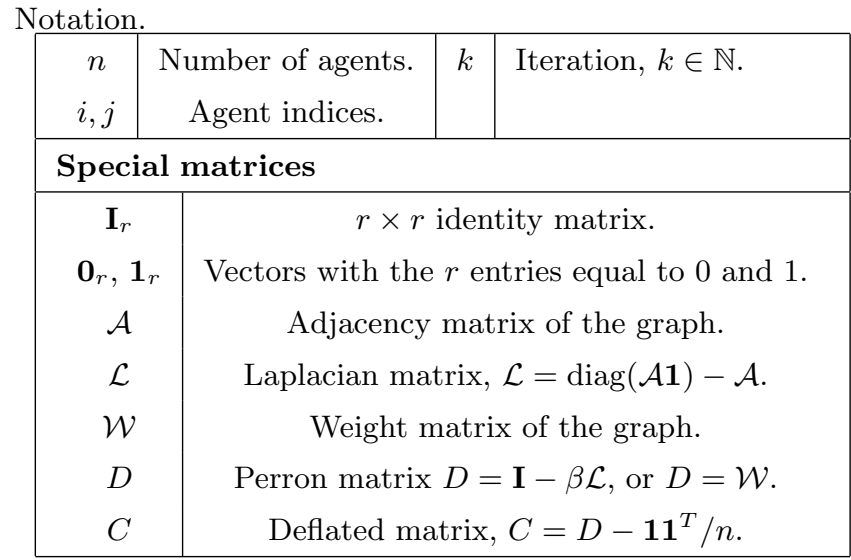

$\mathcal{L}$. This algebraic connectivity plays an important role in the continuous-time multi-agent dynamics. For instance, it dominates the convergence rate of consensus algorithms [10], while in event-triggered consensus [15] it is a crucial parameter for the agents to decide when to trigger. Equivalently, several discrete-time distributed algorithms rely on weight matrices $\mathcal{W}$ compatible with the undirected graph $\mathcal{G}$ which are symmetric, doublystochastic $\mathcal{W} \mathbf{1}=\mathbf{1}, \mathbf{1}^{T} \mathcal{W}=\mathbf{1}^{T}$; and that satisfy $\mathcal{W}_{i i}>0$ and for $j \neq i, \mathcal{W}_{i j}=0$ if $j \notin \mathcal{N}_{i}$ and $\mathcal{W}_{i j}>0$ otherwise. Examples of these weight matrices include expressions that depend on the Laplacian, $\mathcal{W}=\mathbf{I}-\beta \mathcal{L}$, or the widely used Metropolis weights [19] among others. For these systems, the convergence properties and convergence speed depend on the essential spectral radius $\rho_{\text {ess }}(\mathcal{W})$

$$
\rho_{\text {ess }}(\mathcal{W})=\rho\left(\mathcal{W}-\mathbf{1 1}^{T} / n\right)
$$

For instance, the classical discrete-time average consensus method $\mathbf{x}(k+1)=\mathcal{W} \mathbf{x}(k)$, has the following convergence rate $\|\mathbf{e}(k)\| /\|\mathbf{e}(0)\| \leq \rho_{\text {ess }}(\mathcal{W})^{k}$, where $\mathbf{e}(k)=$ $\mathbf{x}(k)-\mathbf{1 1}^{T} \mathbf{x}(0) / n$.

It is common for higher level algorithms to establish conditions on their behavior or on they parameters such as $\alpha_{1}<\lambda_{\star}(\mathcal{L})$, or $\alpha_{2}>\rho_{\text {ess }}(\mathcal{W})$. Usually, algebraic connectivity methods, have asymptotic convergence, i.e., if we let $\hat{\lambda}_{i}(k)$ be the estimated algebraic connectivity after $k$ iterations of the algorithm, then $\lim _{k \rightarrow \infty} \hat{\lambda}_{i}(k)=$ $\lambda_{\star}(\mathcal{L})$, but for a finite $k$, we have $\hat{\lambda}_{i}(k) \neq \lambda_{\star}(\mathcal{L})$. If we do not know how $\hat{\lambda}_{i}(k)$ approaches $\lambda_{\star}(\mathcal{L})$, i.e., if $\hat{\lambda}_{i}(k) \lesseqgtr$ $\lambda_{\star}(\mathcal{L})$, the selection of the number of steps $k$ for which we should execute the algebraic connectivity estimation method, and the adjust of a parameter $\alpha_{1}$ satisfying $\alpha_{1}<\lambda_{\star}(\mathcal{L})$, are non trivial. Instead, if we now that our estimate approaches $\lambda_{\star}(\mathcal{L})$ satisfying $\hat{\lambda}_{i}(k) \leq \lambda_{\star}(\mathcal{L})$ for all $k$, then we can just choose $\alpha_{1}<\hat{\lambda}_{i}(k) \leq \lambda_{\star}(\mathcal{L})$ at any step $k$.

Problem 2.1 (Laplacian Matrices) Our goal is to

\begin{tabular}{|c|c|}
\hline \multicolumn{2}{|c|}{ Matrix operations, eigenvalues and eigenvectors } \\
\hline$A_{i j},[A]_{i j}$ & $(i, j)$ entry of matrix $A$. \\
$\operatorname{diag}\left(b_{1}, \ldots, b_{r}\right)$ & matrix $A$ with $A_{i i}=b_{i}$ and $A_{i j}=0$. \\
$\lambda_{i}(A)$ & $i^{\text {th }}$ eigenvalue of $A$. \\
$\mathbf{v}_{i}(A)$ & $i^{\text {th }}$ eigenvector of $A$. \\
$\lambda_{\star}(\mathcal{L})$ & Algebraic connectivity. \\
$\rho_{\text {ess }}(\mathcal{W})$ & Essential spectral radius. \\
$\|A\|_{\infty}$ & Induced $\infty$-norm, $\max _{i} \sum_{j=1}^{n}\left|A_{i j}\right|$. \\
$\|A\|_{2}$ & Spectral norm, $\max _{i} \sqrt{\lambda_{i}\left(A^{T} A\right)}$. \\
$\rho(A)$ & Spectral radius, $\max _{i}\left|\lambda_{i}(A)\right|$. \\
\hline
\end{tabular}

design distributed algorithms to allow the agents to compute $\lambda_{\star}(\mathcal{L})$, and/or a lower bound of $\lambda_{\star}(\mathcal{L})$ in a distributed fashion.

Problem 2.2 (Weight Matrices) Our goal is to design distributed algorithms to allow the agents to compute the essential spectral radius $\rho_{\text {ess }}(\mathcal{W})$, and/or an upper bound of $\rho_{\text {ess }}(\mathcal{W})$ in a distributed fashion.

Next we focus on the estimation of the algebraic connectivity. Later, Section 4 explains the adaptations for estimating the essential spectral radius instead.

The Power Iteration method [6] is a popular way to compute the leading eigenvalue and associated eigenvector for a matrix $C$. This method thus can be used for obtaining the algebraic connectivity of $\mathcal{L}$ by letting $C$ be the following deflated version of the Perron matrix of the Laplacian $\mathcal{L},[1,10,18,20]$

$$
C=\mathbf{I}-\beta \mathcal{L}-\mathbf{1 1 ^ { T }} / n,
$$

where the eigenvalues of $C$ and $\mathcal{L}$ are related by

$$
\lambda_{1}(C)=0, \quad \lambda_{i}(C)=1-\beta \lambda_{i}(\mathcal{L}), \text { for } i \in\{2, \ldots, n\},
$$

so that the spectral radius $\rho(C)$ of $C$ is associated to the algebraic connectivity $\lambda_{\star}(\mathcal{L})$ by

$$
\lambda_{\star}(\mathcal{L})=(1-\rho(C)) / \beta, \quad \text { if } 0<\beta<1 / \lambda_{n}(\mathcal{L}) .
$$

From now on, we let $D$ be the not-deflated matrix,

$$
D=\mathbf{I}-\beta \mathcal{L}, \text { so that } \quad C=D-\mathbf{1 1}^{T} / n .
$$

Algorithm 2.3 (Centralized Power Iteration) Let $\mathbf{z}(k) \in \mathbb{R}^{n}$ be an estimate of the leading eigenvector, initialized with any value, and updated at each step $k$ with

$$
\begin{aligned}
& \mathbf{z}(k+1)=C \mathbf{z}(k) /\|C \mathbf{z}(k)\| \\
& \quad=\left(D \mathbf{z}(k)-\mathbf{1 1}^{T} \mathbf{z}(k) / n\right) /\left\|D \mathbf{z}(k)-\mathbf{1 1}^{T} \mathbf{z}(k) / n\right\| .
\end{aligned}
$$


The estimate $\hat{\rho}_{\text {cpi }}(k)$ of the leading eigenvalue $\rho(C)$ at step $k$ is given by the Rayleigh quotient, and the estimated algebraic connectivity $\hat{\lambda}_{c p i}(k)$ is as in eq. (4),

$$
\hat{\rho}_{c p i}(k)=\frac{\mathbf{z}(k)^{T} C \mathbf{z}(k)}{\mathbf{z}(k)^{T} \mathbf{z}(k)}, \quad \hat{\lambda}_{c p i}(k)=\frac{1-\hat{\rho}_{c p i}(k)}{\beta} .
$$

Symbol $\|$.$\| in eq. (6) denotes a vector norm. In fact,$ the same $\hat{\rho}_{c p i}(k), \hat{\lambda}_{c p i}(k)$ are obtained regardless of the specific values used for normalizing.

This method upper bounds $\lambda_{\star}(\mathcal{L})$ whereas our goal was to obtain a lower bound (Problem 2.1), i.e., if we consider the estimate after a finite number of steps $k$, the method believes that the network has a connectivity slightly higher that the actual one.

Lemma 2.4 The algebraic connectivity $\hat{\lambda}_{c p i}(k)$ estimated with Algorithm 2.3 for a symmetric matrix $C$ is related to the true Laplacian algebraic connectivity $\lambda_{\star}(\mathcal{L})$ as follows, for all $k \geq 0$ :

$$
\hat{\lambda}_{c p i}(k) \geq \lambda_{\star}(\mathcal{L})
$$

PROOF. Consider $\hat{\rho}_{c p i}(k)$ in eq. (7),

$$
\hat{\rho}_{c p i}(k)=\frac{\left\|C^{\frac{1}{2}} \mathbf{z}(k)\right\|_{2}^{2}}{\|\mathbf{z}(k)\|_{2}^{2}} \leq \frac{\left\|C^{\frac{1}{2}}\right\|_{2}^{2}\|\mathbf{z}(k)\|_{2}^{2}}{\|\mathbf{z}(k)\|_{2}^{2}}=\left\|C^{\frac{1}{2}}\right\|_{2}^{2},
$$

and for symmetric matrices, $\|C\|_{2}=\rho(C)$, and

$$
\left\|C^{\frac{1}{2}}\right\|_{2}^{2}=\rho\left(C^{1 / 2}\right)^{2}=\rho(C),
$$

giving $\hat{\rho}_{c p i}(k) \leq \rho(C)$, and thus

$$
\hat{\lambda}_{c p i}(k)=\left(1-\hat{\rho}_{c p i}(k)\right) / \beta \geq(1-\rho(C)) / \beta=\lambda_{\star}(\mathcal{L}) .
$$

The distributed implementations of the power iteration method let each agent $i$ maintain one entry $\mathbf{z}_{i}(k)$ of the state vector $\mathbf{z}(k)$. The operations that require global knowledge in eq. (6), i.e., normalization $\left(/\left\|D \mathbf{z}(k)-\mathbf{1 1}^{T} \mathbf{z}(k) / n\right\|\right)$ and deflation $\left(-\mathbf{1 1}^{T} \mathbf{z}(k) / n\right)$, are usually replaced with averaging iterations. Examples include $[14,20]$ for continuous-time systems, and [4] for discrete-time systems. Each agent $i$ updates its component $\mathbf{z}_{i}(k)$ using a slightly different estimated average value, introducing errors in the system. A recent interesting variation of the distributed power iteration for discrete-time systems is given by [11]. The deflation step $\left(-\mathbf{1 1}^{T} \mathbf{z}(k) / n\right)$ in eq. (6) is avoided by building an initial vector $\mathbf{z}(0)$ with zero average. Vector $\mathbf{z}(k)$ is normalized (eq. (6)) using the infinity norm $\left(/\|D \mathbf{z}(k)\|_{\infty}\right)$, which is computed with a max-consensus algorithm [17] that converges in finite time (less than $\operatorname{diam}(\mathcal{G})$ iterations).

\section{Distributed Computation of the Algebraic Connectivity}

We present an distributed method for estimating the algebraic connectivity $\lambda_{\star}(\mathcal{L})$ of an undirected graph, which is not only convergent but that also provides lower and upper bounds at each step $k$. It relies on the observation that $(i)$ the induced infinite norm of a matrix $\|C\|_{\infty}$ can be easily computed in a distributed fashion with a max - consensus method, provided that each node knows a row of this matrix; and that $(i i)\left\|C^{k}\right\|_{\infty}^{\frac{1}{k}}$ successively approaches the spectral radius $\rho(C)$. We will use this method to compute the spectral radius of the deflated matrix $C$. Recall that this matrix $C$ was related to Laplacian $\mathcal{L}$ by (eq. (4))

$$
\begin{aligned}
C & =\mathbf{I}-\beta \mathcal{L}-\mathbf{1 1}^{T} / n, \\
\lambda_{\star}(\mathcal{L}) & =(1-\rho(C)) / \beta, \text { for } \quad 0<\beta<1 / \lambda_{n}(\mathcal{L}) .
\end{aligned}
$$

The method consist of agents computing the induced $\infty$-norm $\|.\|_{\infty}$ of matrix $C^{k}$, which is the maximum absolute row sum of the matrix,

$$
\left\|C^{k}\right\|_{\infty}=\max _{i \in \mathcal{V}}\left\{\left|\left[C^{k}\right]_{i 1}\right|+\cdots+\left|\left[C^{k}\right]_{i n}\right|\right\}
$$

which can be easily obtained by the agents using a distributed max - consensus algorithm, provided that each agent $i$ knows the $i-t h$ row of $C^{k}$. For any induced norm, in particular for the $\infty$-norm, it holds [5, Chap. 5.6]

$$
\rho(C) \leq\|C\|_{\infty}, \text { and } \rho(C)=\lim _{k \rightarrow \infty}\left\|C^{k}\right\|^{\frac{1}{k}},
$$

and for symmetric matrices, i.e., undirected graphs, in addition it holds that [5, Chap. 5.6]

$$
\begin{gathered}
\rho(C)=\|C\|_{2}=\left\|C^{k}\right\|_{2}^{\frac{1}{k}}, \text { and } \\
(\sqrt{n})^{-1}\left\|C^{k}\right\|_{\infty} \leq\left\|C^{k}\right\|_{2},
\end{gathered}
$$

being $\|.\|_{2}$ the spectral norm. These properties allow us to give lower and upper bounds for our estimated algebraic connectivity at each step.

First, we present the distributed method for computing powers of a matrix which is compatible with the graph. Recall that our matrix $C$ is not compatible with the graph. We will use this method to compute the powers $D^{k}$ of matrix $D$ in eq. (5),

$$
D=\mathbf{I}-\beta \mathcal{L}, \text { so that } \quad C=D-\mathbf{1 1}^{T} / n,
$$


and we will later discuss the relationship between the powers of $D$ and $C$. The following distributed power matrix computation method can be used with both symmetric and not symmetric matrices $D$. Our discussion refers to fixed graphs, although the method can be easily extended to time-varying graphs. We only impose the assumption that each agent $i$ has assigned a unique identifier $I D(i)$, e.g., its IP address.

Algorithm 3.1 (Distributed Power Matrix Computation) Each node $i \in \mathcal{V}$ maintains a set of node identifiers $l_{i}(k)$, and an estimate $\hat{D}_{i j}(k)$ of the $(i, j)$ entries of the $k$-th power of $D,\left[D^{k}\right]_{i j}$, associated to the nodes $j$ such that $I D(j) \in l_{i}(k)$.

1: At $k=0$, each node $i \in \mathcal{V}$ initializes a single variable $\hat{D}_{i i}(k)$ and a single identifier,

$$
\hat{D}_{i i}(0)=1, \quad l_{i}(0)=\{I D(i)\}
$$

and sends this data to its neighbors $\mathcal{N}_{i}$.

2: At each step $k \geq 1$, node $i$ first looks for new nodes in the information $l_{j}(k)$ received from its neighbors, and updates its identifiers $l_{i}(k)$ accordingly,

$$
l_{i}(k+1)=\bigcup_{j \in \mathcal{N}_{i} \cup\{i\}} l_{j}(k) .
$$

3: Then, node $i$ creates a new variable $\hat{D}_{i j}(k)$ initialized with $\hat{D}_{i j}(k)=0$, for each recently discovered node $j$,

$$
I D(j) \in l_{i}(k+1) \text { and } I D(j) \notin l_{i}(k) .
$$

4: Finally, node $i$ updates all its variables $\hat{D}_{i j}(k)$,

$$
\hat{D}_{i j}(k+1)=\sum_{j^{\prime} \in \mathcal{N}_{i} \cup\{i\}, I D(j) \in l_{j^{\prime}}(k)} D_{i j^{\prime}} \hat{D}_{j^{\prime} j}(k),
$$

for $I D(j) \in l_{i}(k+1)$, and sends to its neighbors these variables $\hat{D}_{i j}(k+1)$ and the identifiers $l_{i}(k+1)$.

\begin{abstract}
Algorithm 3.2 (Distributed Algebraic Connectivity) Let $\varepsilon \in(0,1)$ a number pre-given to the agents, and $\beta=\varepsilon /(2 n)$. Consider the agents execute Algorithm 3.1 for computing the powers of matrix $D=\mathbf{I}-\beta \mathcal{L}$. Then, at each step $k \geq 1$, each node $i \in \mathcal{V}$ has variables $\hat{D}_{i j}(k)$, for $I D(j) \in l_{i}(k)$, containing the $(i, j)$ entries of the $k$-th power of matrix $D,\left[D^{k}\right]_{i j}$.
\end{abstract}

At each step $k$, each node $i$ computes

$$
c_{i}(k)=\sum_{I D(j) \in l_{i}(k)}\left|\hat{D}_{i j}(k)-1 / n\right|+\left(n-\left|l_{i}(k)\right|\right) / n,
$$

and runs a $\max -$ consensus [17] to get $\max _{j \in \mathcal{V}} c_{j}(k)$. The algebraic connectivity $\hat{\lambda}_{i}(k)$ estimated by each agent $i \in \mathcal{V}$ at step $k \geq 1$ is given by

$$
\hat{\lambda}_{i}(k)=\left(1-\hat{\rho}_{i}(k)\right) / \beta, \quad \hat{\rho}_{i}(k)=\left(\max _{j \in \mathcal{V}} c_{j}(k)\right)^{\frac{1}{k}} .
$$

Observe that this computation of $c_{i}(k)$ in eq. (17) is local to each node $i$, since it maintains the $i-t h$ row of $C^{k}$. Note that the estimated spectral radius $\hat{\rho}_{i}(k)$ associated to step $k$ is available at the nodes $\operatorname{diam}(\mathcal{G})$ iterations later (at iteration $k+\operatorname{diam}(\mathcal{G})$ ). However, the $\max -$ consensus iterations are executed independently (in parallel) to the Algorithm 3.1. This means that agents do not have to wait $\operatorname{diam}(\mathcal{G})$ iterations for the $\max$-consensus to converge before executing a new iteration of Algorithm 3.1.

Now, we discuss the properties of this distributed algebraic connectivity estimation algorithm.

Proposition 3.3 When $D$ is compatible with the graph, (i) the outcomes $\hat{D}_{i j}(k)$ of Algorithm 3.1 at step $k \geq 0$, with $I D(j) \in l_{i}(k)$, are exactly the entries of the $k-t h$ power of $D, D^{k}$; and (ii) for $I D(j) \notin l_{i}(k)$, the entries of the $k$-th power of $D, D^{k}$, equal zero, $\left[D^{k}\right]_{i j}=0$.

PROOF. We first prove (ii). Note that $l_{i}(k)$ contains the identifiers of the nodes which are at $k$ or less hops far from node $i$. If for a particular node $j$ it happens that $I D(j) \notin l_{i}(k)$, then there is no path between nodes $i$ and $j$ with length smaller than or equal to $k$. Since matrix $D$ is compatible with the graph, then the associated entry of the $k$-th power of $D$ equals zero, $\left[D^{k}\right]_{i j}=0$.

Now we focus in (i). For $k=0$, it is straightforward to see that it is true, since $D^{0}=\mathbf{I}$, and $\hat{D}_{i i}=1$ (eq. (14)). For $k \geq 1$, we consider the explicit expression for $D^{k+1}=$ $D \bar{D}^{k}$, and take into account that, since $D$ is compatible with the graph, then $D_{i j^{\prime}}=0$ for $j^{\prime} \notin \mathcal{N}_{i} \cup\{i\}$. Besides, from (ii), $\left[D^{k}\right]_{j^{\prime} j}=0$ when $I D(j) \notin l_{i}(k)$. Thus, each $(i, j)$ entry of $D^{k+1}$ is

$$
\begin{aligned}
{\left[D^{k+1}\right]_{i j} } & =\sum_{j^{\prime}=1}^{n} D_{i j^{\prime}}\left[D^{k}\right]_{j^{\prime} j}=\sum_{j^{\prime} \in \mathcal{N}_{i} \cup\{i\}} D_{i j^{\prime}}\left[D^{k}\right]_{j^{\prime} j} \\
& =\sum_{j^{\prime} \in \mathcal{N}_{i} \cup\{i\}, I D(j) \in l_{j^{\prime}}(k)} D_{i j^{\prime}}\left[D^{k}\right]_{j^{\prime} j},
\end{aligned}
$$

which is the update rule for $\hat{D}_{i j}(k+1)$ in eq. (16).

Algorithm 3.1 provides each agent $i$ with all the entries of the $i-t h$ row of the power matrix $D^{k}$, and only requires each node $i$ to have a unique identifier $I D(i)$. The 
results presented so far hold for both fixed and timevarying graphs. Each node $i$ updates its variables using only its own and its neighbors' data; it stores $n$ scalars, and exchanges $n$ scalars at each iteration $k$. Our algorithm exactly computes $D^{k}$ at each step $k$ (not an estimate of it). Observe that it remains valid if the communication graph is time-varying, and thus $D$ is not fixed but depends on the step $k, D(k)$, in which case each agent $i$ computes

$$
\hat{D}_{i j}(k)=[D(k) D(k-1) \ldots D(0)]_{i j}, \text { for } j \in \mathcal{V}
$$

Remark 3.4 For fixed graphs, the previous method can be used for obtaining the number of nodes n. For each node $i \in \mathcal{V}$, let $k_{i}$ be the first instant for which $l_{i}(k)=l_{i}(k-1)$,

$$
k_{i}=\min \left\{k \mid l_{i}(k)=l_{i}(k-1)\right\} .
$$

Then, $n=\left|l_{i}\left(k_{i}\right)\right|$. Note that $l_{i}(k-1)$ contains the identifiers of the $(k-1)$-hop neighbors of $i$. By the definition of a path, if there are no new nodes at distance $k$, then there cannot be new nodes at distances greater than $k$. Therefore $l_{i}(k-1)$ contains the identifiers of all the nodes that are connected with $i$. Since the graph is connected, these nodes are all the nodes in the network and $n=\left|l_{i}\left(k_{i}\right)\right|$. Thus, for fixed graphs, the previous condition can be used to improve the network usage of Algorithm 3.1. Since the first time $l_{i}(k)=l_{i}(k-1)$, agent $i$ can stop executing steps 2 : to 3 : and exchanging variables $l_{i}(k)$.

Algorithm 3.1 can be used for computing powers of a matrix $D$ which is compatible with the graph. Recall, however, that we are interested in computing the spectral radius of matrix $C$, since it is related to the Laplacian algebraic connectivity $\lambda_{\star}(\mathcal{L})$ and to the essential spectral radius $\rho_{\text {ess }}(\mathcal{W})$ of the weigh matrix $\mathcal{W}$ (eq. $\left.(9)\right)$. This matrix $C$ is not compatible with the graph due to the term $11^{T} / n$. Next, we analyze the relationship between the powers $D^{k}, C^{k}$, of matrices $D$ and $C=D-\mathbf{1 1}^{T} / n$ (eq. (13)).

Proposition 3.5 Consider a symmetric Laplacian matrix $\mathcal{L}$, or a symmetric stochastic weigh matrix $\mathcal{W}$, and let $D$ be defined as $D=\mathbf{I}-\beta \mathcal{L}$, or $D=\mathcal{W}$, with $\beta>0$, and $C$ be $C=D-\mathbf{1 1}^{T} / n$. The $k-t h$ powers of matrices $C$ and $D$ are related as follows, for all $k \geq 1$,

$$
C^{k}=\left(D-\mathbf{1 1}^{T} / n\right)^{k}=D^{k}-\mathbf{1 1}^{T} / n
$$

PROOF. Note that, since $\mathbf{1} / \sqrt{n}$ is the eigenvector $\mathbf{v}_{1}(C)$ of $C$ associated to the eigenvalue $\lambda_{1}(C)=0$, then $D=C+\mathbf{1 1}^{T} / n$ and $C$ have the same eigenvectors $\mathbf{v}_{i}(C)=\mathbf{v}_{i}(D)$ for all $i \in\{1, \ldots, n\}$, and all the eigenvalues equal but the first one, $\lambda_{i}(C)=\lambda_{i}(D)$ for all $i \in\{2, \ldots, n\}$, and $\lambda_{1}(C)=0$ whereas $\lambda_{1}(D)=1$. Thus, their $k$-th powers have the following expressions:

$$
\begin{aligned}
& C^{k}=(0)^{k} \mathbf{1 1}^{T} / n+\sum_{i=2}^{n}\left(\lambda_{i}(C)\right)^{k} \mathbf{v}_{i}(C) \mathbf{v}_{i}(C)^{T}, \\
& D^{k}=(1)^{k} \mathbf{1 1}^{T} / n+\sum_{i=2}^{n}\left(\lambda_{i}(C)\right)^{k} \mathbf{v}_{i}(C) \mathbf{v}_{i}(C)^{T},
\end{aligned}
$$

where $0^{k}=0$ and $1^{k}=1$, so that we obtain eq. (20).

Therefore, we let the robots compute powers of this matrix $D$ (each robot $i$ maintains the $i$-th row, $\left.\left[D^{k}\right]_{i 1}, \ldots,\left[D^{k}\right]_{i n}\right)$. When robot $i$ wants to compute instead the $i$-th row of $C^{k}$, it just has to subtract $1 / n$ from each of its elements $\left[D^{k}\right]_{i j}$, for $j \in\{1, \ldots, n\}$.

Theorem 3.6 Let each node $i$ execute Algorithm 3.2 with $\mathcal{G}$ undirected and connected. As $k \rightarrow \infty$, the variables $\hat{\lambda}_{i}(k)$ converge to the Laplacian algebraic connectivity $\lambda_{*}(\mathcal{L})$ for all $i \in \mathcal{V}$,

$$
\lim _{k \rightarrow \infty} \hat{\lambda}_{i}(k)=\lambda_{*}(\mathcal{L}),
$$

and for all $i \in \mathcal{V}$ and all $k$ we have lower and upper bounds for $\lambda_{\star}(\mathcal{L})$ :

$$
\hat{\lambda}_{i}(k) \leq \lambda_{\star}(\mathcal{L}) \leq n^{\frac{-1}{2 k}} \hat{\lambda}_{i}(k)+\left(1-n^{\frac{-1}{2 k}}\right) / \beta .
$$

PROOF. First note that $\beta=\varepsilon /(2 n)$ satisfies $0<\beta<$ $1 / \lambda_{n}(\mathcal{L})$ since $\varepsilon \in(0,1)$ and $\lambda_{n}(\mathcal{L}) \leq 2 d_{\max }<2 n$, where $d_{\text {max }}$ is the maximum degree in the graph. Therefore, the algebraic connectivity is related to the spectral radius of matrix $C$ as in eq. $(9), \lambda_{*}(\mathcal{L})=(1-\rho(C)) / \beta$. From Proposition 3.3, for all $i \in \mathcal{V}$, the variables $\hat{D}_{i j}(k)$ are equal to $\left[D^{k}\right]_{i j}$ for $I D(j) \in l_{i}(k)$, whereas $\left[D^{k}\right]_{i j}=0$ for $I D(j) \notin l_{i}(k)$. Linking this with Proposition 3.5 yields

$$
\begin{aligned}
& {\left[C^{k}\right]_{i j}=\left[D^{k}\right]_{i j}-1 / n, \text { for } I D(j) \in l_{i}(k),} \\
& {\left[C^{k}\right]_{i j}=-1 / n, \text { for } I D(j) \notin l_{i}(k), \text { for all } i \in \mathcal{V}, k \geq 1 .}
\end{aligned}
$$

$c_{i}(k)$ in eq. (17) is the absolute $i-$ th row sum of $C^{k}$, and $\hat{\rho}_{i}(k)$ in eq. (18) equals $\left\|C^{k}\right\|_{\infty}^{\frac{1}{k}}$. For any induced norm, in particular for the $\infty$-norm, it holds [5, Chap. 5.6],

$$
\begin{aligned}
& \rho(C)=\lim _{k \rightarrow \infty}\left\|C^{k}\right\|_{\infty}^{\frac{1}{k}}=\lim _{k \rightarrow \infty} \hat{\rho}_{i}(k), \\
& \rho(C) \leq\|C\|_{\infty},
\end{aligned}
$$

and thus

$$
\rho(C)=\left(\rho\left(C^{k}\right)\right)^{\frac{1}{k}} \leq\left\|C^{k}\right\|_{\infty}^{\frac{1}{k}}=\hat{\rho}_{i}(k) .
$$


Since $C$ is symmetric, its spectral norm $\|C\|_{2}=$ $\max _{i} \sqrt{\lambda_{i}\left(C^{2}\right)}$ equals its spectral radius $\rho(C)=$ $\max _{i}\left|\lambda_{i}(C)\right|$,

$$
\rho(C)=\|C\|_{2}=\left\|C^{k}\right\|_{2}^{\frac{1}{k}}
$$

The spectral $\left\|C^{k}\right\|_{2}$ and induced infinite $\left\|C^{k}\right\|_{\infty}$ norms of $C^{k}$ are related by $(\sqrt{n})^{-1}\left\|C^{k}\right\|_{\infty} \leq\left\|C^{k}\right\|_{2},[5$, Chap. 5.6], which combined with eq. (25) gives

$$
n^{\frac{-1}{2 k}} \hat{\rho}_{i}(k)=n^{\frac{-1}{2 k}}\left\|C^{k}\right\|_{\infty}^{\frac{1}{k}} \leq\left\|C^{k}\right\|_{2}^{\frac{1}{k}}=\rho(C) .
$$

From eqs. (23)-(26),

$$
\begin{aligned}
(\sqrt{n})^{\frac{-1}{k}} \hat{\rho}_{i}(k) & \leq \rho(C) \leq \hat{\rho}_{i}(k), \\
\rho(C) & =\lim _{k \rightarrow \infty} \hat{\rho}_{i}(k),
\end{aligned}
$$

which combined with eqs. (18) and (4) gives eqs. (21) and (22).

In fact, we can show that the convergence rate of the considered algorithm is no slower than $O(1 / k)$.

Corollary 3.7 Let each node $i$ execute Algorithm 3.2 with $\mathcal{G}$ undirected and connected. The estimation errors, containing the difference between the estimated $\hat{\lambda}_{i}(k)$ and the true Laplacian algebraic connectivity $\lambda_{*}(\mathcal{L})$, evolve according to:

$$
\left|\lambda_{*}(\mathcal{L})-\hat{\lambda}_{i}(k)\right| \leq \frac{1}{k} \log \sqrt{n}\left(1 / \beta-\lambda_{*}(\mathcal{L})\right)+O\left(1 / k^{2}\right)
$$

PROOF. Noticing

$$
\begin{aligned}
\left|\lambda_{*}(\mathcal{L})-\hat{\lambda}_{i}(k)\right| & \leq\left(\sqrt{n}^{\frac{1}{k}}-1\right)\left(1 / \beta-\lambda_{*}(\mathcal{L})\right) \\
& =\left(e^{\frac{1}{k} \log \sqrt{n}}-1\right)\left(1 / \beta-\lambda_{*}(\mathcal{L})\right),
\end{aligned}
$$

the desired conclusion becomes clear immediately in light of the Taylor series of $e^{\frac{1}{k} \log \sqrt{n}}-1$ with respect to $1 / k$.

We finally discuss some issues regarding the number of nodes $n$. Note that the number of nodes $n$ is used in the computation of $\beta$. In case the agents do not know $n$ from the beginning, they can compute $\beta=\varepsilon /\left(2 d_{\max }\right)$, which satisfies $\beta<1 / \lambda_{n}(\mathcal{L})$ as in eq. (4) by executing a max - consensus algorithm on the nodes degrees in an initial phase. Once $\beta$ has been computed, agents can start Algorithm 3.2. At each step $k$ of Algorithm 3.2, agents can always execute Algorithm 3.1 for computing the powers of matrix $D=\mathbf{I}-\beta \mathcal{L}$. However, they can only execute eqs. (17)-(18) for getting the output $\hat{\lambda}_{i}(k)$ when they know $n$. At each step $k$ agents use eq. (19) to find out if they have already found $n$ and thus if they can proceed with eqs. (17)-(18). Alternatively, $n$ can be computed in an initial phase.

\section{Estimation of the Essential Spectral Radius}

The algorithm proposed (Section 3) can be also used for computing the essential spectral radius $\rho_{\text {ess }}(\mathcal{W})$ of the weight matrix $\mathcal{W}$ by replacing matrix $C$ in eqs. (3), (9) with the following deflated version $C$ of the weight ma$\operatorname{trix} \mathcal{W}$

$$
C=\mathcal{W}-\mathbf{1 1}^{T} / n, \text { so that } \quad \rho_{\text {ess }}(\mathcal{W})=\rho(C),
$$

and replacing matrix $D$ in eqs. (5), (13) with the notdeflated version of this matrix $C$,

$$
D=\mathcal{W}, \text { so that } \quad C=D-\mathbf{1 1}^{T} / n
$$

Since $\rho_{\text {ess }}(\mathcal{W})=\rho(C)$, then the essential spectral radius estimated at each step $k$ and agent $i$ equals the spectral radius estimated with the algorithm, i.e., $\hat{\rho}_{c p i}(k)$ in eq. (7) for Algorithm 2.3; and $\hat{\rho}_{i}(k)$ in eq. (18) for the Distributed Algebraic Connectivity method proposed in this paper (Algorithm 3.2).

The results discussed in this paper can be easily adapted as well to the essential spectral radius case as follows. Lemma 2.4 reads $\hat{\rho}_{c p i}(k) \leq \rho(C)$, which is the opposed behavior to our goal (Problem 2.2). Theorem 3.6 states that for all $i \in \mathcal{V}$,

$$
\lim _{k \rightarrow \infty} \hat{\rho}_{i}(k)=\rho_{\text {ess }}(\mathcal{W}),
$$

and for all $i \in \mathcal{V}$ and all $k$ we have lower and upper bounds for $\rho_{\text {ess }}(\mathcal{W})$ :

$$
(\sqrt{n})^{\frac{-1}{k}} \hat{\rho}_{i}(k) \leq \rho_{e s s}(\mathcal{W}) \leq \hat{\rho}_{i}(k) .
$$

Finally, Corollary 3.7 states that the estimation errors, containing the difference between the estimated $\hat{\rho}_{i}(k)$ and the true essential spectral radius $\rho_{\text {ess }}(\mathcal{W})$ of the weight matrix, evolve according to

$$
\left|\hat{\rho}_{i}(k)-\rho_{e s s}(\mathcal{W})\right| \leq \frac{1}{k} \log \sqrt{n} \rho_{e s s}(\mathcal{W})+O\left(\frac{1}{k^{2}}\right) .
$$

\section{Simulations}

We have performed a set of simulations with $n=20$ nodes placed as in the two scenarios in Fig. 1. We evaluate the computation of both the algebraic connectivity 


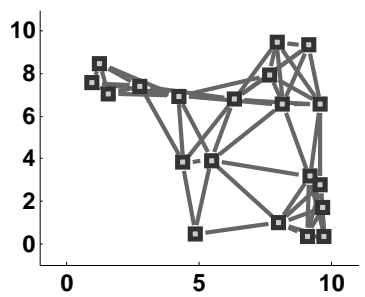

(a) Random graph

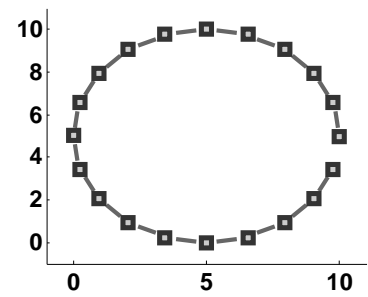

(b) String graph
Fig. 1. Scenarios tested. (a): 20 agents (black squares) are placed randomly in a region of $10 \times 10$ meters; there is an edge $e=(i, j) \in \mathcal{E}$ (gray lines) between pairs of agents that are closer than 4 meters; $\lambda_{\star}(\mathcal{L})=0.7103$ and $\rho_{\text {ess }}(\mathcal{W})=0.9187$. (b): string graph with agents 1 and 20 in the extremes; $\lambda_{\star}(\mathcal{L})=0.0246$ and $\rho_{\text {ess }}(\mathcal{W})=0.9918$.

$\lambda_{\star}(\mathcal{L})$ of the Laplacian $\mathcal{L}$, and the essential spectral radius $\rho_{\text {ess }}(\mathcal{W})$ of the Metropolis [19] weight matrix $\mathcal{W}$.

The Distributed Algebraic Connectivity method (Section 3, Algorithm 3.2) is executed using the graphs in Fig. 1. The estimated algebraic connectivity $\hat{\lambda}_{i}(k)$ at step $k$ (Fig. 2, dac, dark red dashed) is the same for all the agents $i \in \mathcal{V}$; it lower bounds the true algebraic connectivity $\lambda_{*}(\mathcal{L})$ (black solid), and asymptotically converges to $\lambda_{*}(\mathcal{L})$. The expression $(\sqrt{n})^{\frac{-1}{k}} \hat{\lambda}_{i}(k)+\left(1-(\sqrt{n})^{\frac{-1}{k}}\right) / \beta$ (dac up, red solid) upper bounds $\lambda_{*}(\mathcal{L})$, and asymptotically converges to $\lambda_{*}(\mathcal{L})$. The rates of convergence for the random and string graphs are very similar.

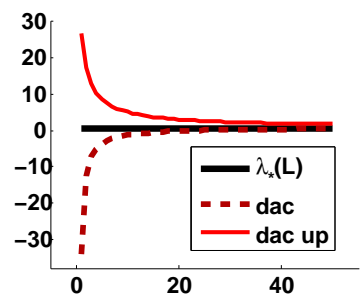

(a) Random graph

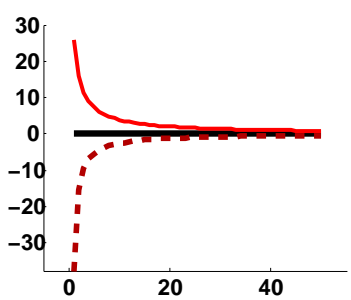

(b) String graph
Fig. 2. Algebraic connectivity $\hat{\lambda}_{i}(k)$ estimated for 40 steps of the Distributed Algebraic Connectivity (Algorithm 3.2). $\hat{\lambda}_{i}(k)$ (dac, dark red dashed) and the expression $(\sqrt{n})^{\frac{-1}{k}} \hat{\lambda}_{i}(k)+\left(1-(\sqrt{n})^{\frac{-1}{k}}\right) / \beta$ (dac up, red solid) respectively lower and upper bound the true algebraic connectivity $\lambda_{*}(\mathcal{L})$ (black solid), and both converge to $\lambda_{*}(\mathcal{L})$.

Algorithm 3.2 is used for computing the essential spectral radius $\rho_{\text {ess }}(\mathcal{W})$ of the weight matrices for the graphs in Fig. 1. The estimated essential spectral radius $\hat{\rho}_{i}(k)$ at step $k$ (Fig. 3, dac, dark red dashed) is the same for all the agents $i \in \mathcal{W}$; it upper bounds the true essential spectral radius $\rho_{\text {ess }}(\mathcal{W})$ (black solid), and asymptotically converges to $\rho_{\text {ess }}(\mathcal{W})$. The expression $(\sqrt{n})^{\frac{-1}{k}} \hat{\rho}_{i}(k)$ (dac low, red solid) lower bounds $\rho_{\text {ess }}(\mathcal{W})$, and asymptotically converges to $\rho_{\text {ess }}(\mathcal{W})$. For the two graphs, the convergence rate is very similar.

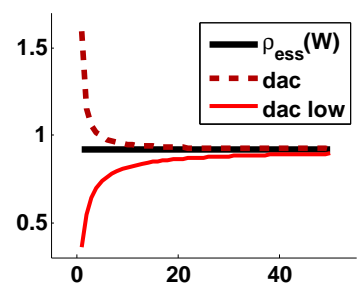

(a) Random graph

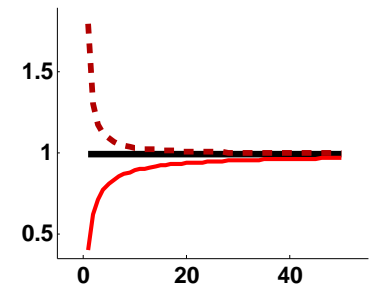

(b) String graph
Fig. 3. Essential spectral radius $\hat{\rho}_{i}(k)$ estimated for 40 steps of the Distributed Algebraic Connectivity (Algorithm 3.2). $\hat{\rho}_{i}(k)$ (dac, dark red dashed) and the expression $(\sqrt{n})^{\frac{-1}{k}} \hat{\rho}_{i}(k)$ (dac low, red solid) respectively upper and lower bound the true essential spectral radius $\rho_{\text {ess }}(\mathcal{W})$ (black solid), and both asymptotically converge to $\rho_{\text {ess }}(\mathcal{W})$.

We compare the estimates of our Distributed Algebraic Connectivity method (Algorithm 3.2), and of the Centralized Power Iteration (Algorithm 2.3). Fig. 4 shows the algebraic connectivity $\hat{\lambda}_{c p i}(k)$ (cpi, gray solid) estimated with the Centralized Power Iteration (Algorithm 2.3) with 10 different initial vectors, for the two graphs in Fig. 1. The Centralized Power Iteration converges exponentially (order $r^{k}$, where $r$ is the rate between the two largest modulus eigenvalues). Thus it is expected to exhibit a fast convergence, whereas our method has a convergence rate order $\frac{1}{k}$ (Corollary 3.7). In practice, the Centralized Power Iteration $\hat{\lambda}_{c p i}(k)$ converged to the true $\lambda_{*}(\mathcal{L})$ (black solid) slowly for the string graph, where the two largest modulus eigenvalues have similar values. Besides, the estimates $\hat{\lambda}_{c p i}(k)$ are larger than $\lambda_{*}(\mathcal{L})$, as opposed to the goal stated in Problem 2.1. Our method (dac, dark red dashed) converged fast to $\lambda_{*}(\mathcal{L})$ in both cases, and it produced $\hat{\lambda}_{i}(k)$ smaller than $\lambda_{*}(\mathcal{L})$; additionally, it gives an upper bound in case it is needed.

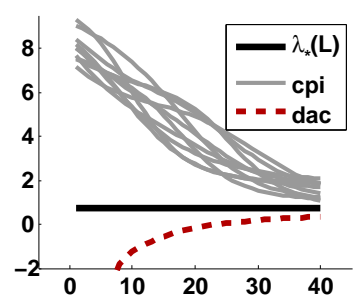

(a) Random graph

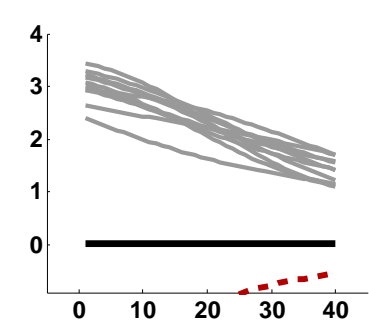

(b) String graph
Fig. 4. Algebraic connectivity estimated along 40 steps of the Centralized Power Iteration (Algorithm 2.3), using 10 different initial vectors $\left(\hat{\lambda}_{c p i}(k), c p i\right.$, gray solid), and along 40 steps of our method $\left(\hat{\lambda}_{i}(k), d a c\right.$, dark red dashed). The true algebraic connectivity $\lambda_{*}(\mathcal{L})$ is shown in black solid.

The distributed implementations of the power iteration method let each agent $i$ maintain one entry $\mathbf{z}_{i}(k)$ of the state vector $\mathbf{z}(k)$. At each iteration, the agents 
send constant size messages for updating the state vector. This is a benefit compared to our proposal, where messages have size $n$. Fig. 5 analyzes the evolution of the estimates of the algorithms versus the total size of messages sent per agent. We have compared the estimates $\hat{\lambda}_{i}(k)$ (dac, dark red dashed) of our Distributed Algebraic Connectivity method (Algorithm 3.2), against two distributed power iteration methods. In the first one $\hat{\lambda}_{a p i_{i}}(k)$ (api, blue solid), the operations that require global knowledge in eq. (6), i.e., normalization $\left(/\left\|D \mathbf{z}(k)-\mathbf{1 1}^{T} \mathbf{z}(k) / n\right\|\right)$ and deflation $\left(-\mathbf{1 1}^{T} \mathbf{z}(k) / n\right)$, are replaced with $T_{\text {cons }}=\{20,50,100\}$ Average Consensus iterations, using the discrete-time rule $\mathbf{w}(t+1)=W \mathbf{w}(t)$, with $W$ the Metropolis weight matrix [19]. As $T_{\text {cons }}$ increases, $\hat{\lambda}_{a p i_{i}}(k)$ (api, blue solid) becomes closer to the Centralized Power Iteration estimates $\hat{\lambda}_{c p i}(k)$ (cpi, gray dashed), and converges to a value closer to $\lambda_{*}(\mathcal{L})$ (black solid), although the communication consumption increases as well. Our estimates $\hat{\lambda}_{i}(k)$ (dac, dark red dashed) converge faster, i.e., using less messages, to the true algebraic connectivity $\lambda_{\star}(\mathcal{L})$ (black solid). The second distributed power iteration tested [11] avoids the deflation step $\left(-\mathbf{1 1}^{T} \mathbf{z}(k) / n\right)$ in eq. (6) by building an initial vector $\mathbf{z}(0)$ with zero average, and it normalizes vector $\mathbf{z}(k)$ (eq. (6)) using the infinity norm $\left(/\|D \mathbf{z}(k)\|_{\infty}\right)$, which is computed with a max -Consensus algorithm [17] that converges in finite time. The estimates $\hat{\lambda}_{m p i}(k)$ of this distributed power iteration with $\max -$ Consensus method (mpi, green solid) converge to the true algebraic connectivity $\lambda_{\star}(\mathcal{L})$ (black solid), using a similar number of messages as our proposed method $\hat{\lambda}_{i}(k)$ (dac, dark red dashed).

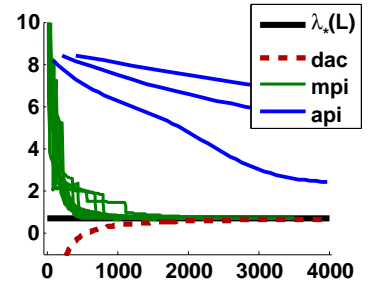

(a) Random graph

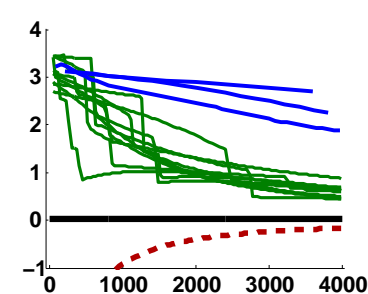

(b) String graph
Fig. 5. Algebraic connectivity (y-axis) estimated by our method $\hat{\lambda}_{i}(k)$ (dac, dark red dashed); by the Dist. Power Iter. with max-Consensus $\left(\hat{\lambda}_{m p i}(k), m p i\right.$, green solid); and by the Dist. Power Iter. with Average Consensus $\left(\hat{\lambda}_{a p i_{i}}(k)\right.$, $a p i$, blue solid), for $i=10$ and $T_{\text {cons }}=\{20,50,100\}$; relative to the size of the messages exchanged ( $x$-axis).

\section{Conclusions}

We have presented a distributed method to compute the algebraic connectivity $\lambda_{\star}(\mathcal{L})$ and the essential spectral radius $\rho_{\text {ess }}(\mathcal{W})$ for networked agent systems with limited communication. At each iteration, the algorithm produces both an upper and a lower bound estimates of $\lambda_{\star}(\mathcal{L})$. We have proved theoretically and experimentally that both estimates asymptotically converge to the true $\lambda_{\star}(\mathcal{L})$. This ability to give upper and lower bounds has a great importance for combining this method with higher level algorithms, executing both processes simultaneously. Although our agents send messages of size $n$ at each step, we have shown that our method has similar communication load as distributed implementations of the Power Iteration method.

\section{Acknowledgements}

Partially supported by the Spanish projects and grants Ministerio de Ciencia e Innovacion DPI2009-08126, DPI2012-32100, BES-2007-14772, by grants from the French program investissement d'avenir managed by the National Research Agency (ANR), the European Commission (Auvergne FEDER funds) and the Région Auvergne in the framework of the LabEx IMobS3 (ANR-10-LABX-16-01), and by the Swedish Research Council through contract VR-2009-3948.

\section{References}

[1] R. Aragues, G. Shi, D. V. Dimarogonas, C. Sagues, and K. H. Johansson. Distributed algebraic connectivity estimation for adaptive event-triggered consensus. In American Control Conference, pages 32-37, Montreal, Canada, June 2012.

[2] D. V. Dimarogonas and K. H. Johansson. Bounded control of network connectivity in multi-agent systems. IET Control Theory and Applications, 4(8):1330 - 1338, 2010.

[3] M. Franceschelli, A. Gasparri, A. Giua, and C. Seatzu. Decentralized laplacian eigenvalues estimation for networked multi-agent systems. In IEEE Conf. on Decision and Control, pages 2717 -2722, Shanghai, P. R. China, December 2009.

[4] M. C. De Gennaro and A. Jadbabaie. Decentralized control of connectivity for multi-agent systems. In IEEE Conf. on Decision and Control, pages 3628 -3633, San Diego, CA, December 2006.

[5] R. A. Horn and C. R. Johnson. Matrix Analysis. Cambridge University Press, Cambridge, UK, 1985.

[6] A.S. Householder. The Theory of Matrices in Numerical Analysis. Dover Publications, New York, 1964.

[7] M. Ji and M. Egerstedt. Distributed coordination control of multiagent systems while preserving connectedness. IEEE Transactions on Robotics, 23(4):693 - 703, 2007.

[8] D. Kempe and F. McSherry. A decentralized algorithm for spectral analysis. Journal of Computer and System Sciences, 74(1):70 - 83, 2008.

[9] E. Montijano, J. I. Montijano, and C. Sagues. Adaptive consensus and algebraic connectivity estimation in sensor networks with chebyshev polynomials. In IEEE Conf. on Decision and Control, pages 4296-4301, Orlando, FL, USA, December 2011.

[10] R. Olfati-Saber, J. A. Fax, and R. M. Murray. Consensus and cooperation in networked multi-agent systems. Proceedings of the IEEE, 95(1):215-233, 2007. 
[11] B. N. Oreshkin, M. J. Coates, and M. G. Rabbat. Optimization and analysis of distributed averaging with short node memory. IEEE Transactions on Signal Processing, 58(5):2850-2865, 2010.

[12] V. M. Preciado, M. M. Zavlanos, A. Jadbabaie, and G. J. Pappas. Distributed control of the laplacian spectral moments of a network. In American Control Conference, pages 4462 -4467, Baltimore, Maryland, USA, July 2010.

[13] Z. Qu, C. Li, and F. Lewis. Cooperative control based on distributed estimation of network connectivity. In American Control Conference, pages 3441 - 2446, San Francisco, CA, USA, July 2011.

[14] L. Sabattini, N. Chopra, and C. Secchi. On decentralized connectivity maintenance for mobile robotic systems. In IEEE Conf. on Decision and Control, pages 988-993, Orlando, FL, USA, December 2011.

[15] G. S. Seyboth, D. V. Dimarogonas, and K. H. Johansson. Event-based broadcasting for multi-agentaverage consensus. Automatica, 49(1):245 - 252, 2013.

[16] A. Simonetto, T. Keviczky, and R. Babuska. On distributed maximization of algebraic connectivity in robotic networks. In American Control Conference, pages 2180 - 2185, San Francisco, CA, USA, July 2011.
[17] A. Tahbaz-Salehi and A. Jadbabaie. A one-parameter family of distributed consensus algorithms with boundary: From shortest paths to mean hitting times. In IEEE Conf. on Decision and Control, pages 4664-4669, San Diego, CA, USA, December 2006.

[18] L. Xiao and S. Boyd. Fast linear iterations for distributed averaging. Systems \& Control Letters, 53:65-78, 2004.

[19] L. Xiao, S. Boyd, and S. Lall. A space-time diffusion scheme for peer-to-peer least-square estimation. In Symposium on Information Processing of Sensor Networks (IPSN), pages 168 - 176, Nashville, TN, April 2006.

[20] P. Yang, R.A. Freeman, G.J. Gordon, K.M. Lynch, S.S. Srinivasa, and R. Sukthankar. Decentralized estimation and control of graph connectivity for mobile sensor networks. Automatica, 46(2):390 - 396, 2010.

[21] M. M. Zavlanos and G. J. Pappas. Controlling connectivity of dynamic graphs. In IEEE Conf. on Decision and Control, pages 6388 -6393, Seville, Spain, December 2005.

[22] M. M. Zavlanos and G. J. Pappas. Distributed connectivity control of mobile networks. IEEE Transactions on Robotics, 24(6):1416 -1428, December 2008. 